\title{
A Probabilistic Approach to Computerized Tracking of Arterial Walls in Ultrasound Image Sequences
}

\author{
Baris Kanber ${ }^{1}$ and Kumar Vids Ramnarine ${ }^{2}$ \\ ${ }^{1}$ Department of Cardiovascular Sciences, University of Leicester, Clinical Sciences Wing, Glenfield General Hospital, \\ Leicester LE3 9QP, UK \\ ${ }^{2}$ Department of Medical Physics, University Hospitals of Leicester NHS Trust, Leicester Royal Infirmary, \\ Sandringham Building, Leicester LE1 5WW, UK \\ Correspondence should be addressed to Baris Kanber, bk20@le.ac.uk
}

Received 30 October 2012; Accepted 19 November 2012

Academic Editors: Y.-S. Chen, E. Ciaccio, and C. S. Lin

Copyright ( 2012 B. Kanber and K. V. Ramnarine. This is an open access article distributed under the Creative Commons Attribution License, which permits unrestricted use, distribution, and reproduction in any medium, provided the original work is properly cited.

\begin{abstract}
Tracking of arterial walls in ultrasound image sequences is useful for studying the dynamics of arteries. Manual delineation is prohibitively labour intensive and existing methods of computerized segmentation are limited in terms of applicability and availability. This paper presents a probabilistic approach to the computerized tracking of arterial walls that is effective and easy to implement. In the probabilistic approach, given a point $\mathrm{B}$ with a probability $P_{\mathrm{b}}$ of being in an arterial lumen of interest, the probability $P_{\mathrm{a}}$ that a neighbouring point A is also a part of the same lumen is proportional to $P_{\mathrm{b}}$ with a Gaussian fall in probability with increasing grayscale contrast between the two points. Efficacy of the probabilistic algorithm was evaluated by testing it on ultrasound images and image sequences of the carotid arteries and the abdominal aorta and various laboratory, ultrasound test objects. The results showed that the probabilistic algorithm produced robust and effective lumen segmentation in the majority of cases encountered. Comparison with a conventional region growing technique based on intensity thresholding with a running, regional intensity average identified the main benefits of the probabilistic approach as increased immunity to speckle noise within the arterial lumen and a reduced susceptibility to region overflowing at boundary imperfections.
\end{abstract}

\section{Introduction}

Greyscale ultrasound imaging (B-mode) is an established tool for the noninvasive imaging of the human body. Such imaging procedures are often accompanied by measurements that are conveniently performed using the ultrasonic calipers. However, it becomes a time-consuming manual task for the operator if the measurements need to be repeated a large number of times, for example, over a time series. In B-Mode vascular ultrasound, such a situation arises when one needs to track the position of the arterial walls over many frames in order to study to distension of the arteries throughout the cardiac cycle. Although specific solutions for tracking the position of the arterial walls using B-Mode ultrasound are available (Table 1), for example, by region tracking/block matching [1] or computerized edge detection [2], many of these techniques are limited in terms of applicability and some techniques have particular vulnerability to image noise. Also, a general purpose segmentation algorithm should be able to track the position of the arterial walls over a sizeable length of the artery and for any vessel orientation and morphology.

One solution to the problem of arterial wall tracking was by Wendelhag et al. [3] who described a method for measuring the intima-media thickness (IMT) and the lumen diameter for the carotid and femoral arteries by means of an analysis system based on dynamic programming [4]. However, it was reported that manual corrections were required in a significant portion of the images. Beux et al. [5] presented an automatic procedure to study the endothelium-dependent dilation of the brachial artery which involved imposing a threshold on the normalized greyscale intensity to locate the arterial lumen and an intensity gradient criterion to subsequently locate the arterial lumen boundaries. However, 
TABLE 1: A selection of specific solutions reported to date applicable to the problem of tracking the vascular walls in B-mode ultrasound image sequences.

\begin{tabular}{|c|c|c|c|}
\hline Reference & Basis of technique & Applications & Limitations \\
\hline$[3]$ & Cost function minimization & $\begin{array}{l}\text { Intima-media thickness (IMT) and arterial } \\
\text { lumen diameter measurement. }\end{array}$ & $\begin{array}{l}\text { Extensive manual corrections were reported } \\
\text { to be required }\end{array}$ \\
\hline$[5]$ & $\begin{array}{l}\text { Greyscale intensity and } \\
\text { gradient thresholding }\end{array}$ & $\begin{array}{l}\text { Endothelium-dependent dilation of the } \\
\text { brachial artery. }\end{array}$ & $\begin{array}{l}\text { Dependence on vessel orientation, } \\
\text { curvature, and appearance }\end{array}$ \\
\hline$[2]$ & Edge detection & $\begin{array}{l}\text { Carotid artery diameter and intima-media } \\
\text { thickness (IMT) measurement. }\end{array}$ & $\begin{array}{l}\text { Operator intervention is frequently needed } \\
\text { during the systolic expansion of the artery } \\
\text { when the arterial wall moves a relatively } \\
\text { large distance between frames }\end{array}$ \\
\hline$[6]$ & Active contours & $\begin{array}{l}\text { Detection of the intima-media complex of } \\
\text { the far wall of the common carotid artery. }\end{array}$ & Long processing times \\
\hline [7] & Artificial neural networks & $\begin{array}{l}\text { Detection of the near and far walls of the } \\
\text { artery in the longitudinal plane. }\end{array}$ & $\begin{array}{l}\text { Relatively horizontal and straight vessel } \\
\text { assumed }\end{array}$ \\
\hline$[1]$ & $\begin{array}{l}\text { Region tracking/block } \\
\text { matching }\end{array}$ & Estimation of carotid artery wall motion. & $\begin{array}{l}\text { Limited number of points can be tracked } \\
\text { due to computational cost and speckle } \\
\text { decorrelation }\end{array}$ \\
\hline [8] & Fast marching algorithm & $\begin{array}{l}\text { Segmentation of intravascular ultrasound } \\
\text { images in 3D. }\end{array}$ & $\begin{array}{l}\text { Required manual delineation of initial } \\
\text { contours close to the arterial lumen } \\
\text { boundaries }\end{array}$ \\
\hline [9] & Hough transform & $\begin{array}{l}\text { Extraction of carotid artery surface in the } \\
\text { longitudinal and transverse planes. }\end{array}$ & $\begin{array}{l}\text { Longitudinal and transverse arterial sections } \\
\text { approximated as straight lines and circles }\end{array}$ \\
\hline$[10]$ & $\begin{array}{l}\text { Polar representation, } \\
\text { grey-level histograms, cost } \\
\text { function minimization, and } \\
\text { probabilistic considerations }\end{array}$ & $\begin{array}{l}\text { Delineation of lumen boundaries in } \\
\text { intravascular ultrasound (IVUS) images. }\end{array}$ & $\begin{array}{l}\text { Specific to IVUS, modeled the lumen surface } \\
\text { as a mixture of Gaussians placing limitations } \\
\text { on the type of contour that can be traced }\end{array}$ \\
\hline$[11]$ & $\begin{array}{l}\text { Edge detection and } \\
\text { mathematical morphology }\end{array}$ & $\begin{array}{l}\text { Delineate vessel lumen boundaries in } \\
\text { transverse cross-sections of the common } \\
\text { carotid artery. }\end{array}$ & $\begin{array}{l}\text { Whether the method is also expected to } \\
\text { work for longitudinal cross sections of } \\
\text { arteries is not known. Also, edge detection } \\
\text { techniques have an inherent vulnerability to } \\
\text { image noise }\end{array}$ \\
\hline
\end{tabular}

this technique worked only in longitudinal cross-sectional views of arteries with specific assumptions made regarding the orientation, curvature, and appearance of the artery. Cheng et al. [6] described a method for detecting the intimamedia complex of the far wall of the common carotid artery using active contour models; however, the processing of a single frame of ultrasound image was reported to have taken between 30 seconds and 1 minute which was a major drawback.

Newey and Nassiri [7] employed artificial neural networks to detect the anterior and posterior vessel walls of arteries in the longitudinal plane, but it was clear that this technique was directly applicable only to relatively straight and horizontal sections of arteries. The technique described by Cardinal et al. [8] for the segmentation of intravascular ultrasound (IVUS) images worked by detecting a mixture of the Rayleigh distributions in the IVUS data followed by a fast marching algorithm which converged on the boundaries of interest; however, the method required the manual delineation of initial contours near the contours of interest to operate. Golemati et al. [9] used the Hough transform (HT) to extract the carotid artery lumen surface from the longitudinal and transverse sections of arteries, but the arterial shapes were modelled as straight lines and circles, respectively, limiting applicability in real-life situations. Mendizabal-Ruiz et al. [10] described a probabilistic segmentation method for IVUS, modeling the lumen contour as a periodic mixture of Gaussians and once again placing constraints on the type of contour that can be tracked. Yang et al. [11] employed edge detection and mathematical morphology techniques to delineate vessel lumen boundaries in transverse cross-sections of the common carotid artery (CCA), but it was not specified whether the method is also expected to work for longitudinal cross-sections.

A recent survey of ultrasound image segmentation methods [12] presented a selection of methodologies for various clinical applications, including intravascular ultrasound; however, segmentation of longitudinal cross-sections of arteries was not covered. Thus, although specific methods for B-Mode ultrasound carotid artery image segmentation have been developed, there appears to be currently no simple solution that can be applied to the diverse range of arterial configurations and imaging conditions encountered and yet be easily implementable by different centres. Also, some techniques, such as those based on edge detection, are particularly vulnerable to speckle noise as the latter 
produces false edge signals. The efficiency of the method is also important as long processing times hinder the practical analysis of long-duration ultrasound image sequences and any possible implementation in real-time.

We have developed a probabilistic approach to segmentation of ultrasound images of the carotid artery and demonstrated good arterial wall tracking, comparable to a more established tissue Doppler imaging technique $[13,14]$. In this paper, we describe our algorithm that effectively segments arterial images in both the longitudinal and transverse crosssections with little effort on the operators' part.

\section{Methods}

In our probabilistic approach, the probabilities of individual neighbouring points being in an arterial lumen were associated using a Gaussian relationship. Given a greyscale ultrasound image, a corresponding probability matrix was evaluated where each matrix element represented the probability of that element's respective image point being in the artery of interest. The probabilities were computed as follows: given a point $\mathrm{B}$ which had a probability $P_{\mathrm{b}}$ of belonging to a certain arterial lumen, the probability $P_{\mathrm{a}}$ that a neighbouring point $\mathrm{A}$ also belonged to the same lumen was evaluated as being directly proportional to $P_{\mathrm{b}}$ with a Gaussian dependence on the greyscale contrast between two points, and an average was taken over all 8 immediate neighbours (1). Here $G_{\mathrm{b}}$ and $G_{\mathrm{a}}$ were the greyscale intensities of points $\mathrm{B}$ and $\mathrm{A}$, and the constant $\zeta$ was determined by considering the amount of greyscale contrast $\left(G_{\mathrm{th}}\right)$ required to reduce $P_{\mathrm{a}}$ to $1 / 2$ that of $P_{\mathrm{b}}$. In the case of multiple seed points, the maximum of the probabilities evaluated using each of the seed points were used

$$
P_{\mathrm{a}}=\left(\frac{1}{8}\right) * \sum P_{\mathrm{b}} \exp \left(-\frac{\left(G_{\mathrm{b}}-G_{\mathrm{a}}\right)^{2}}{\zeta}\right),
$$

where $\zeta=G_{\mathrm{th}}{ }^{2} / \log 2$ and the sum was taken over the 8 immediate neighbours.

The value of $G_{\text {th }}$ was set at $1 \%$ of the full range by default but could be altered by the operator if necessary. The operator provided one or more initial seed points which were assigned a probability of 1 and the probabilities of the remaining points were determined using (1). Images were preprocessed by applying a circular averaging filter of radius $R=5$ pixels.

Efficacy of the algorithm was evaluated by testing it on clinical images of the carotid arteries and the abdominal aorta and various laboratory, ultrasound test objects. Clinical data included B-Mode carotid artery image sequences from patients acquired using a Philips HDI5000 scanner with an L12-5 probe and a Philips iU22 scanner with an L9-3 probe (Philips Healthcare, Eindhoven, The Netherlands). Image sequences were also obtained from a healthy volunteer using an Aixplorer scanner (Supersonic Imagine, Aix-en-Provence, France) with the L15-4 probe. The use of the clinical data for this purpose was approved by the Leicestershire and Rutland Medical Ethics Committee, and patients and the volunteer gave their informed consent to participate in the study.
Laboratory data included ultrasound image sequences from walled and wall-less flow phantoms and various laboratory test objects acquired using the Philips HDI5000 scanner with the L12-5 probe. The tissue mimicking material used in the construction of the hypo- and hyper-echoic test objects, and the wall-less flow phantom was an agarbased formulation [15]. In the flow circuit of the walled (CFLEX, Cole-Pamer, IL, USA) and wall-less flow phantoms, a blood mimicking fluid was circulated $[16,17]$. A computer controlled fluid pump was used to circulate the blood mimicking fluid using a carefully selected input waveform to induce dilations in the phantom similar to the dilation characteristics of a real carotid artery [18].

Performance was also compared against a conventional region growing technique based on intensity thresholding with a running average region intensity [19]. Images were preprocessed using the same filter, and same the seed points and threshold values were used in both the case of the probabilistic algorithm and the conventional region growing technique.

The efficiency of the probabilistic algorithm was also evaluated under high image-noise conditions by means of adding zero-mean, single-sided Gaussian noise of varying strengths to the carotid artery ultrasound images and by testing the method on noisy image sequences from the abdominal aorta and walled and wall-less vessel phantoms (speckle noise within the vessel lumen).

All implementations were carried out using MATLAB (MathWorks, Natick, MA, USA) with portions of the probabilistic algorithm written in the $C$ language to speed up the process. The analyses were performed on a personal computer with a Intel Core i5-2500K CPU (Intel Corporation, $\mathrm{CA}, \mathrm{USA}$ ) running at $3.30 \mathrm{GHz}$. The PC was running the 64bit version of Windows 7 Ultimate (Microsoft Corporation, Washington, DC, USA).

\section{Results}

Setting the algorithm threshold to $2 \%$ and choosing a point inside the arterial lumen in a carotid artery image with atherosclerotic plaque buildup on the far-wall, the probabilistic algorithm produced the first pass estimate boundary outline and the corresponding probability map seen in Figure 1. Adding another seed point produced the overall probability map and segmentation result seen in Figure 2. The final arterial boundary outline including that of the plaque surface obtained by adding three additional seed points was as shown in Figure 3. A close-up view of the segmentation result over the plaque surface can be seen in Figure 4.

The result of tracking the arterial lumen for a longitudinal image sequence with one seed point was as shown in Figure 5. Investigations made on the processing of this 90 frame image sequence of dimensions 263 (height) $\times 256$ (width) pixels indicated a processing time of approximately 33 milliseconds/frame on the analysis computer used. However, since the implementation was not designed to take full advantage of the multicore CPU architecture and was not 


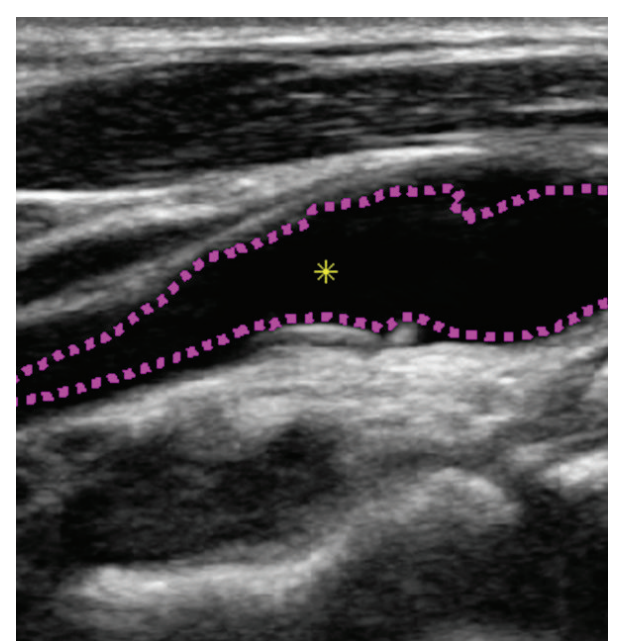

(a)

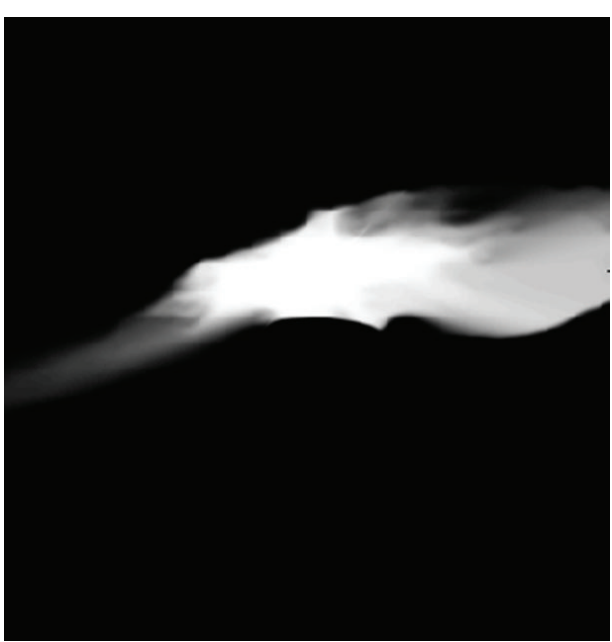

(b)

FIGURE 1: First pass segmentation result (a) for a carotid artery image with plaque buildup on the far wall of the artery. The corresponding probability map (b).

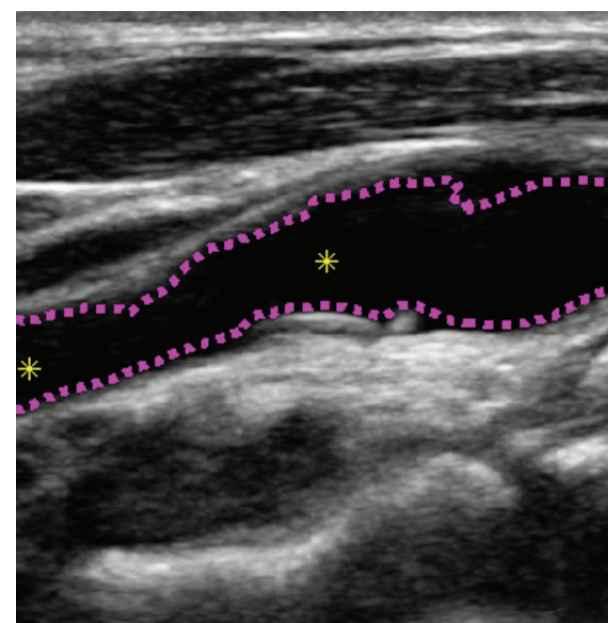

(a)

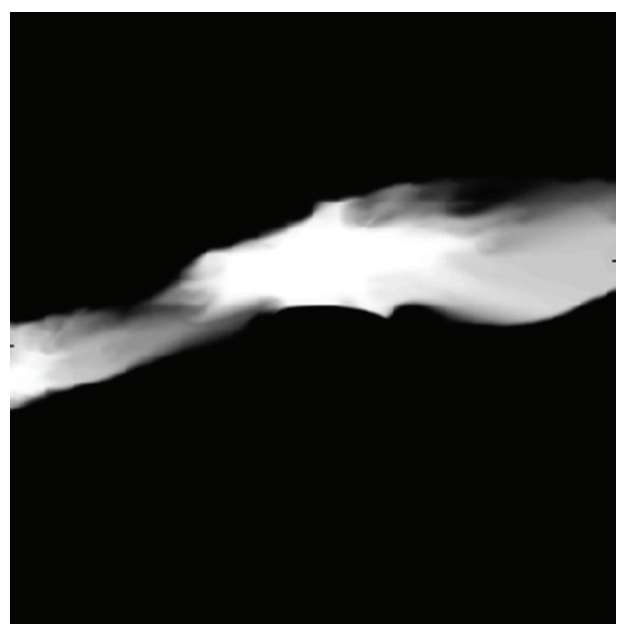

(b)

FIgURE 2: The results of adding another seed point. Segmentation result (a) and combined probability map (b).

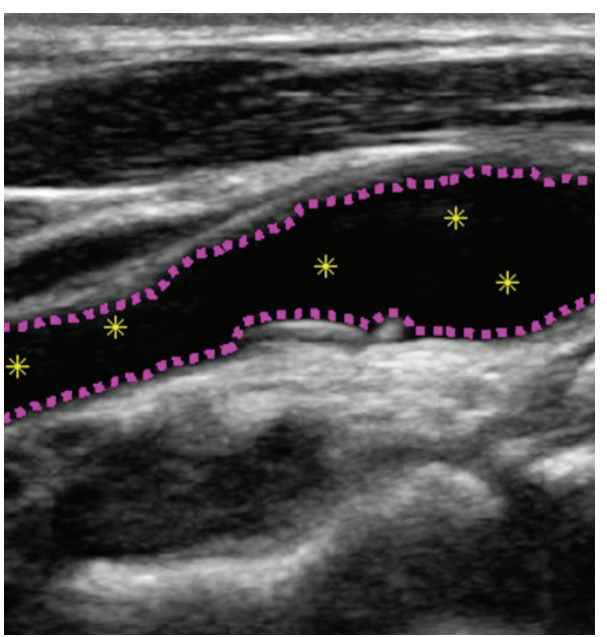

(a)

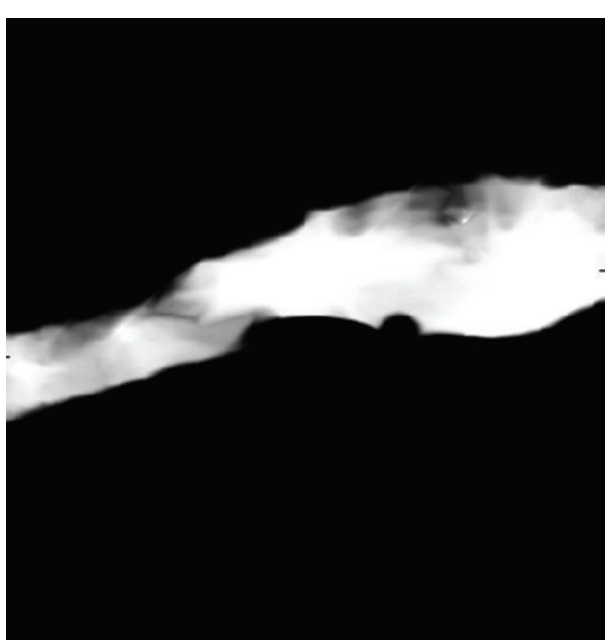

(b)

FIGURE 3: Final segmentation result (a) and probability map (b) with an additional 3 seed points. 


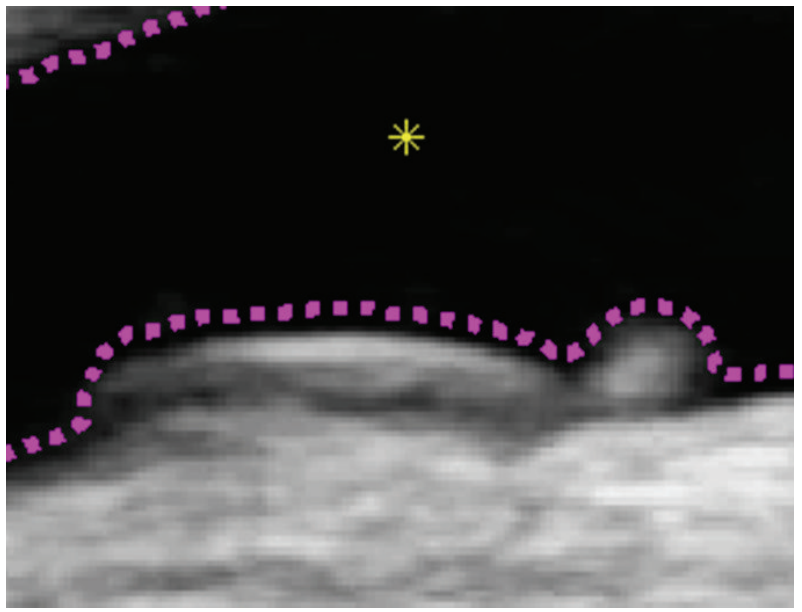

FIGURE 4: A close-up view of the segmentation result over the plaque surface.

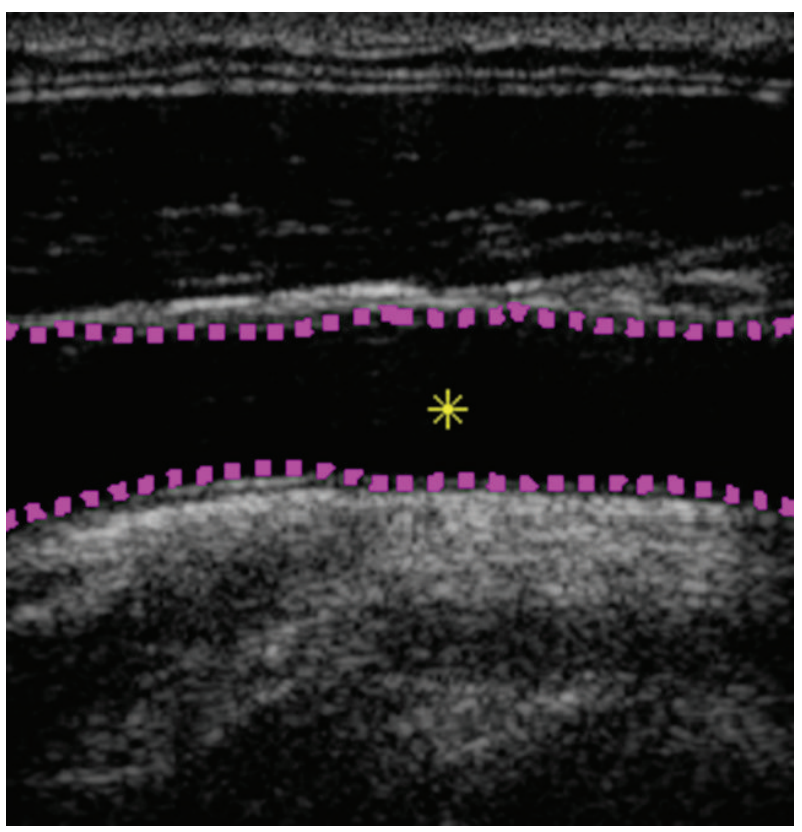

FIGURE 5: Tracking of the arterial lumen for an image sequence (single frame shown). The whole image sequence is available at https://dl .dropbox.com/u/13857734/pp/tt.avi.

optimised, it was also observed that the processor as a whole was only running at approximately $30 \%$ of full capacity during the analysis.

Arterial lumens could be effectively segmented with a few seed points in a variety of arterial configurations and image noise conditions (Figure 6). The result of segmentation and tracking of the residual arterial lumen and plaque surface in the transverse plane was as in Figure 7. Investigation of segmentation performance in the presence of artificially added Gaussian image noise produced the results seen in Figure 8. In an image sequence from the abdominal aorta, wall tracking in the presence of ultrasound artifacts and substantial amounts of image noise was as in Figure 9.
The results of lumen surface tracking for a walled flow phantom with blood mimicking fluid in the flow circuit produced the results in Figure 10. Tracking results for the laboratory test objects and a comparison between Vernier caliper measured and algorithm-determined dimensions were as in Figure 11 and Table 2.

Tracking of the vessel lumen diameter for the common carotid artery from the healthy volunteer using an image sequence obtained on the AIXplorer platform produced the arterial dilation waveform seen in Figure 12.

Comparison of algorithm efficacy against the conventional region growing technique showed that the performance of the probabilistic algorithm surpassed that of the con- 


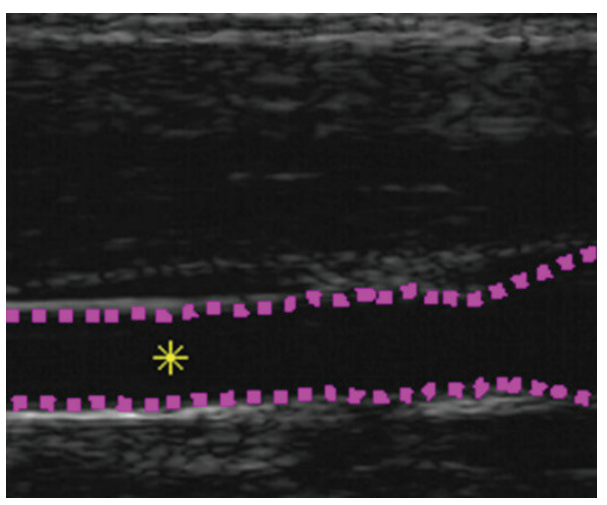

(a)

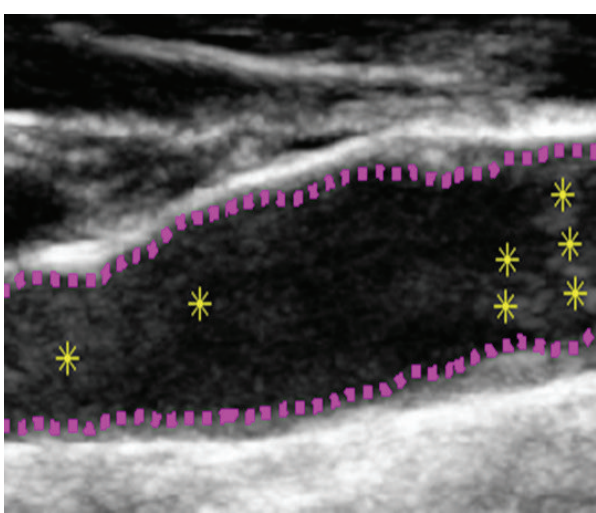

(c)

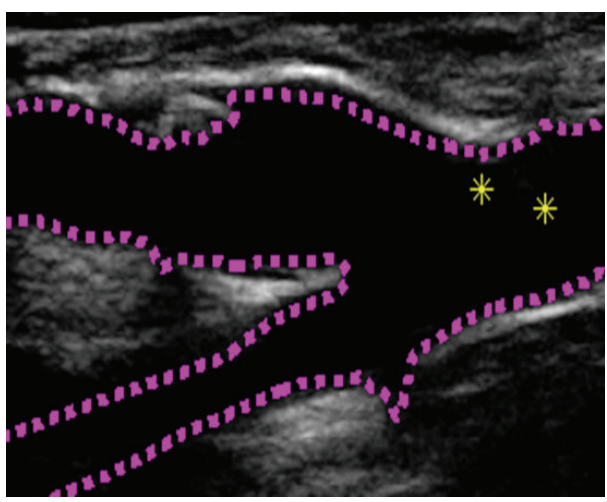

(b)

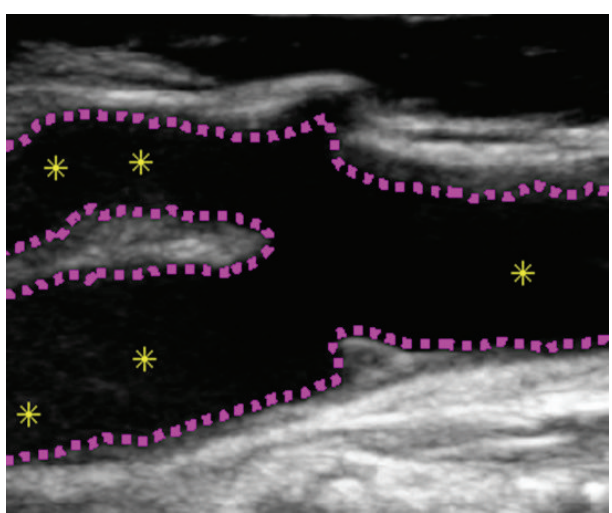

(d)

FIGURE 6: Arterial lumen segmentation in a variety of vessel configurations and image-noise conditions.

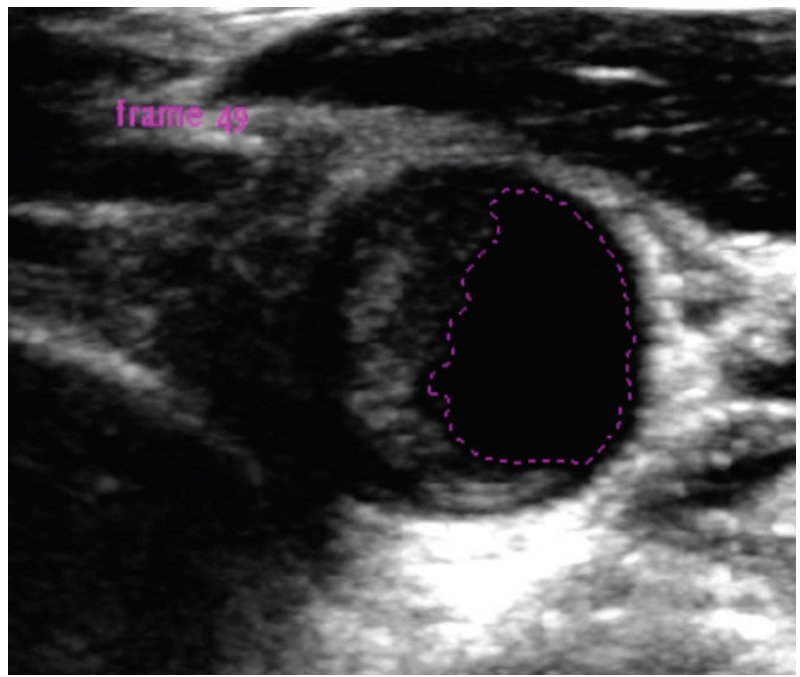

FIGURE 7: Tracking of the residual arterial lumen and plaque surface in the transverse plane (single frame shown). The whole image sequence is available at http://dl.dropbox.com/u/13857734/pp/T1.avi.

ventional region growing algorithm with differences more apparent under high image-noise conditions (Figure 13).

\section{Discussion}

This paper presented a method based on a probabilistic approach that can be used to efficiently segment out and track blood vessel boundaries in B-Mode ultrasound images and image sequences. The results showed that the technique can be used to track arterial lumens simply and efficiently in both the longitudinal and transverse imaging planes, including in the presence of substantial amounts of speckle noise. Boundary segmentation was robust in the presence of significantly strong, artificially added image noise and in 


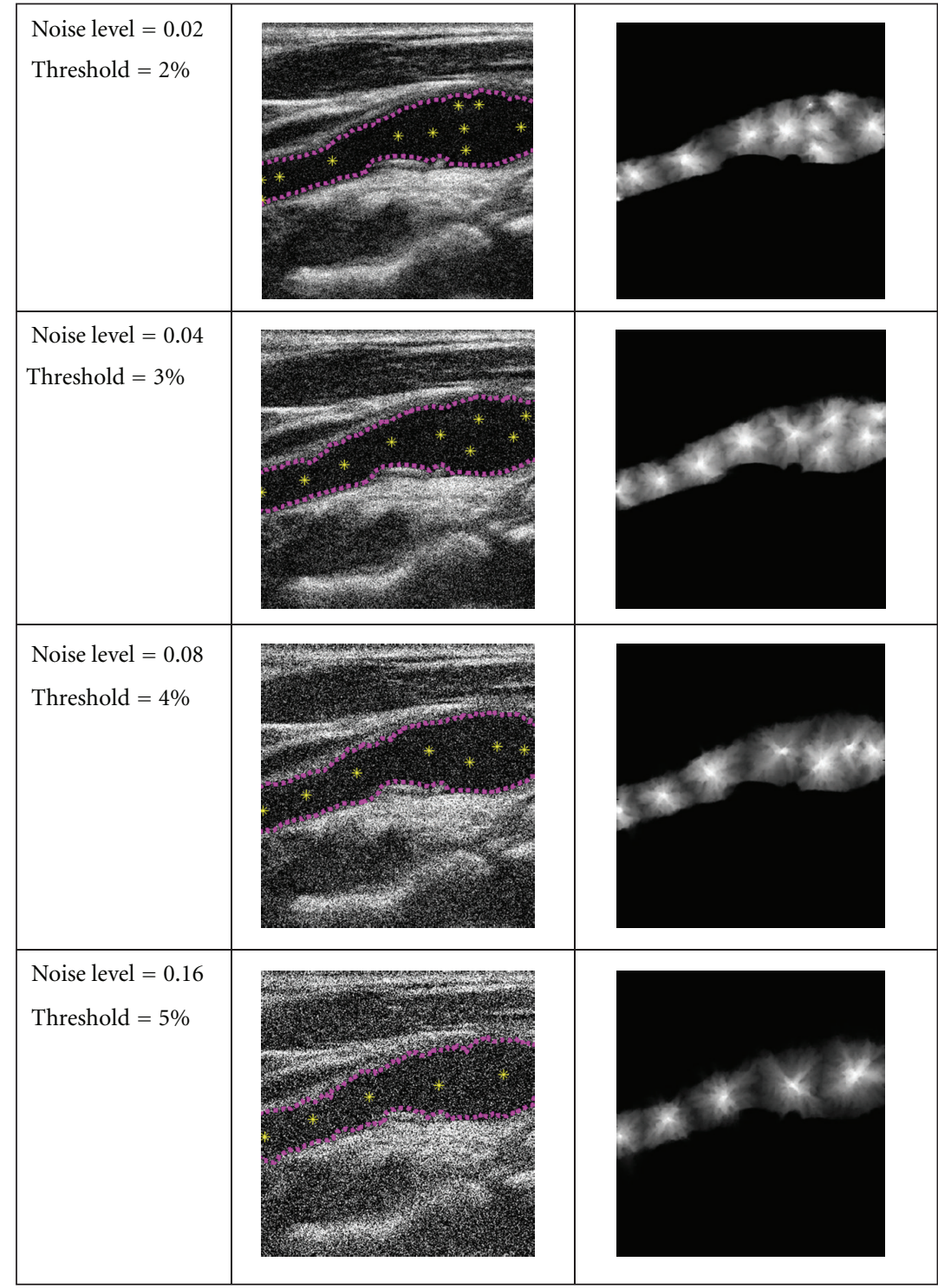

FIGURE 8: Results of segmentation of the arterial lumen and plaque surface in the presence of artificially added Gaussian noise of varying strengths. Each row gives the probability map (right) and segmentation result (middle) for given noise and threshold settings (left). sqrt $($ Noise level $) \times 255$ gives the approximate standard deviation of the Gaussian noise added in grey levels (e.g., noise level $=0.02$ corresponds to a standard deviation of approximately 36.1 grey levels, cf., maximum range of 255 grey levels).

TABLE 2: Comparison between caliper $\left(d_{\text {cal }}\right)$ and algorithm $\left(d_{\mathrm{al}}\right)$ made diameter measurements for the laboratory test objects.

\begin{tabular}{lccc}
\hline Object & Type & $d_{\text {cal }}[\mathrm{mm}]( \pm 0.5 \mathrm{~mm})$ & $d_{\mathrm{al}}[\mathrm{mm}]$ \\
\hline 1 & Hypoechoic & 4.0 & $3.9 \pm 0.2$ \\
8 & Hyperechoic & 9.0 & $8.8 \pm 0.2$ \\
2 & Hypoechoic & 12.0 & $12.4 \pm 0.2$ \\
7 & Hyperechoic & 14.2 & $14.0 \pm 0.3$ \\
3 & Hypoechoic & 24.0 & $24.3 \pm 0.6$ \\
6 & Hyperechoic & 23.2 & $23.4 \pm 0.2$ \\
4 & Hypoechoic & 37.5 & $37.4 \pm 0.2$ \\
5 & Hyperechoic & 39.0 & $38.9 \pm 0.2$ \\
\hline
\end{tabular}

an ultrasound image sequence from the abdominal aorta with ultrasound artifacts. In the case of the walled and wall-less laboratory flow phantoms, good boundary tracking was obtained in the presence of high-intensity reflections within the vessel lumen. Tracking the lumen diameter of the common carotid artery in a healthy volunteer produced a detailed waveform showing the variation of the lumen diameter over the cardiac cycles including the dicrotic notch.

In a separate validation of the method, dimensions of various hyper- and hypoechoic laboratory, ultrasound test objects measured using the probabilistic method were compared with the Vernier caliper measured physical dimensions and similar values were obtained within the error ranges of the measurements. A comparison with a conventional region 


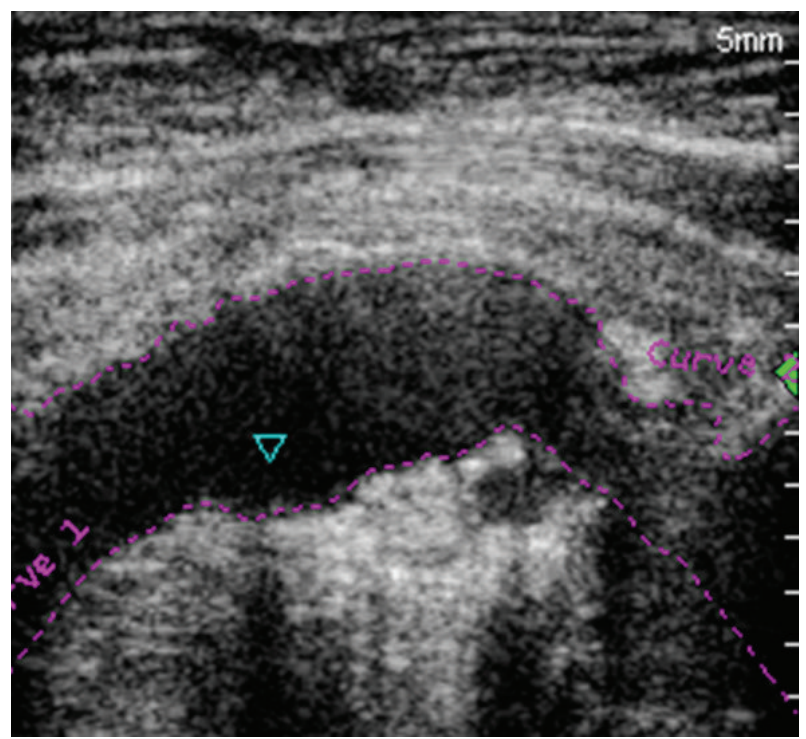

Figure 9: Tracking of the arterial lumen in the abdominal aorta (single frame shown). The whole image sequence is available at http://dl .dropbox.com/u/13857734/pp/AA_1.avi.

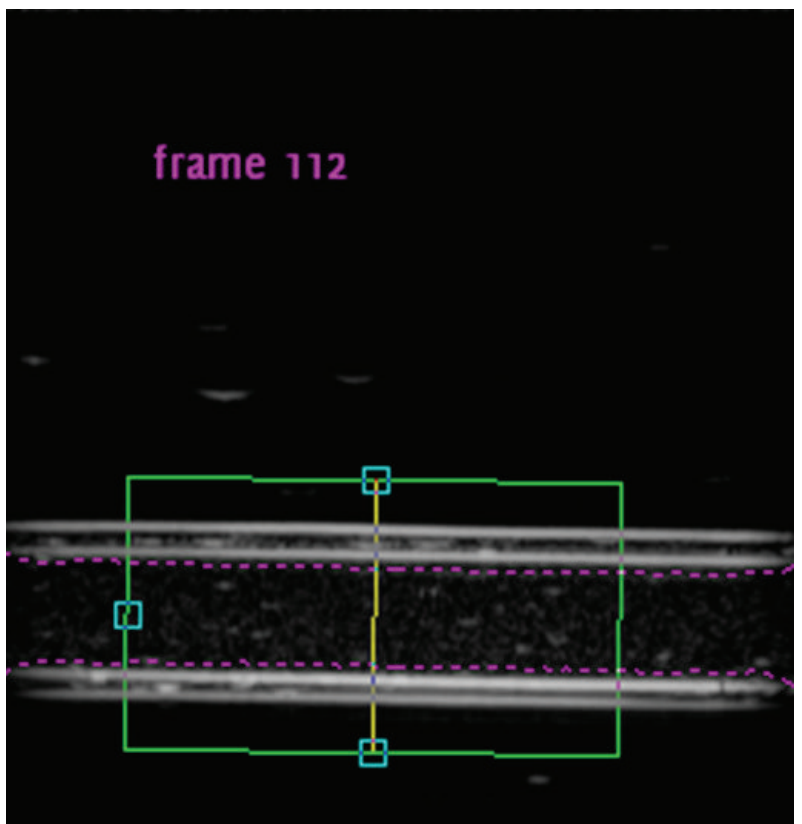

FIGURE 10: Tracking of the lumen surface for a walled flow phantom (single frame shown). The whole image sequence available at http://dl .dropbox.com/u/13857734/pp/WFP_1.avi.

growing algorithm showed that the probabilistic approach had better immunity to noise and less susceptibility to region overflowing at boundary imperfections.

The method described in this paper addresses the limitations of the existing arterial lumen detection techniques based on B-Mode ultrasound image analysis. The limitations of the existing techniques included dependence on vessel orientation, curvature, scan plane, and long processing times. The probabilistic technique was found to be sufficiently efficient to allow practical analysis of long image sequences and make real time implementation feasible. The measured average processing time of $33 \mathrm{~ms} /$ frame per seed point for an image sequence of dimensions similar to a typical carotid artery scan indicated that frame rates as high as $30 \mathrm{~Hz}$ may be achieved in real time even with the highly unoptimized implementation used in the study.

The advantages of the probabilistic method include its simplicity and wide area of applicability. This technique was previously found to have good arterial wall tracking performance, comparable to that of tissue Doppler imaging 


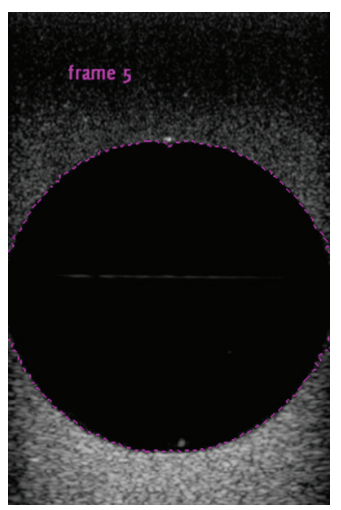

(a)

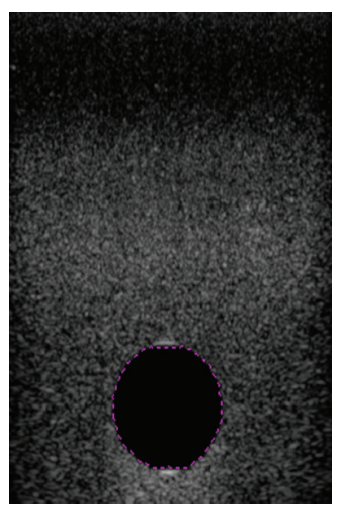

(d)

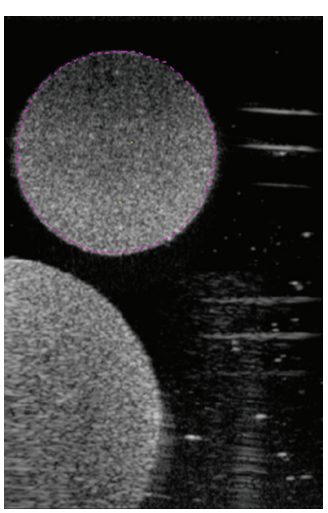

(b)

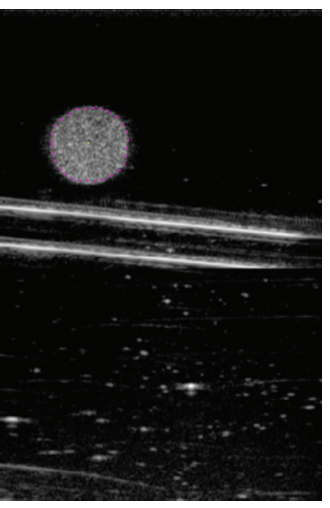

(e)

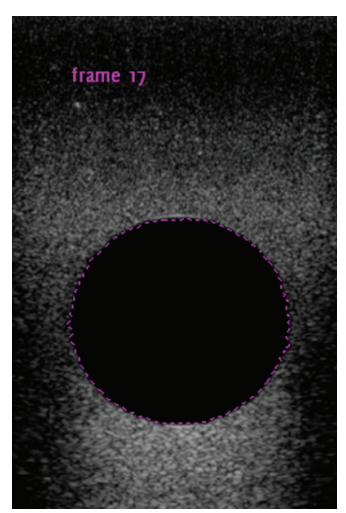

(c)

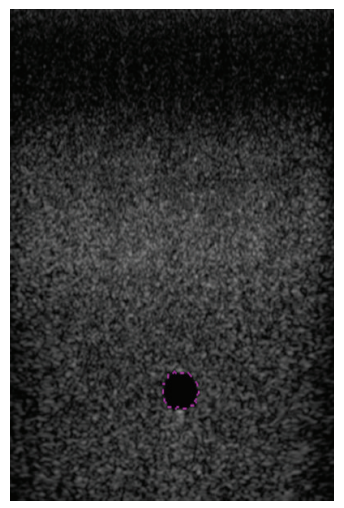

(f)

FIGURE 11: A selection of test results for the delineation of the boundaries of agar based hypo- and hyperechoic test objects.

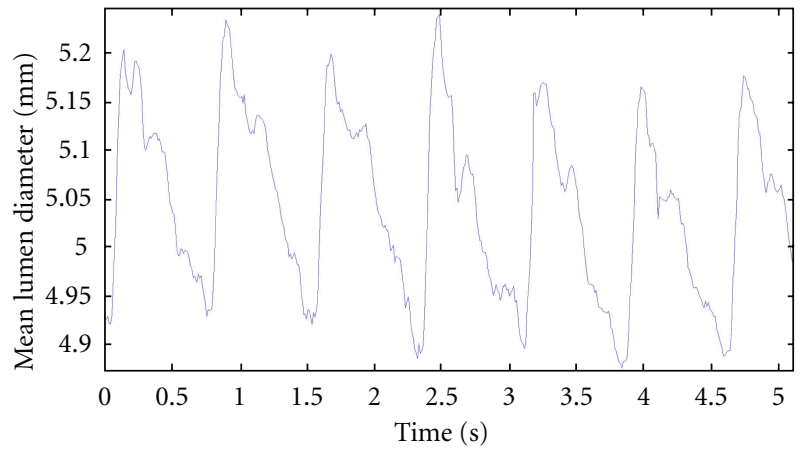

FIGURE 12: Variation of the arterial lumen diameter of the common carotid artery from a healthy volunteer.

[13] which benefits from access to radiofrequency data. The algorithm can be easily adopted in 3 dimensions, and it would be interesting to see what results this would produce in further studies. It is possible that the implementation can be enhanced further, for example, by extending the consideration to texture measures (e.g., local greyscale characteristics).

In conclusion, the method presented in this paper, based on a probabilistic approach to the segmentation of BMode ultrasound carotid artery images, produces robust segmentation results, including in the presence of substantial amounts of image noise, with little effort on the user's part.

\section{Conflict of Interests}

The authors confirm that there is no known conflict of interests associated with this publication and that they do not have any direct or indirect relationship with any commercial identity mentioned in this paper.

\section{Acknowledgments}

The authors are grateful to Professor A. R. Naylor and his team at the Department of Cardiovascular Sciences, particularly Mr. Tim Hartshorne, Chief Vascular Scientist, 


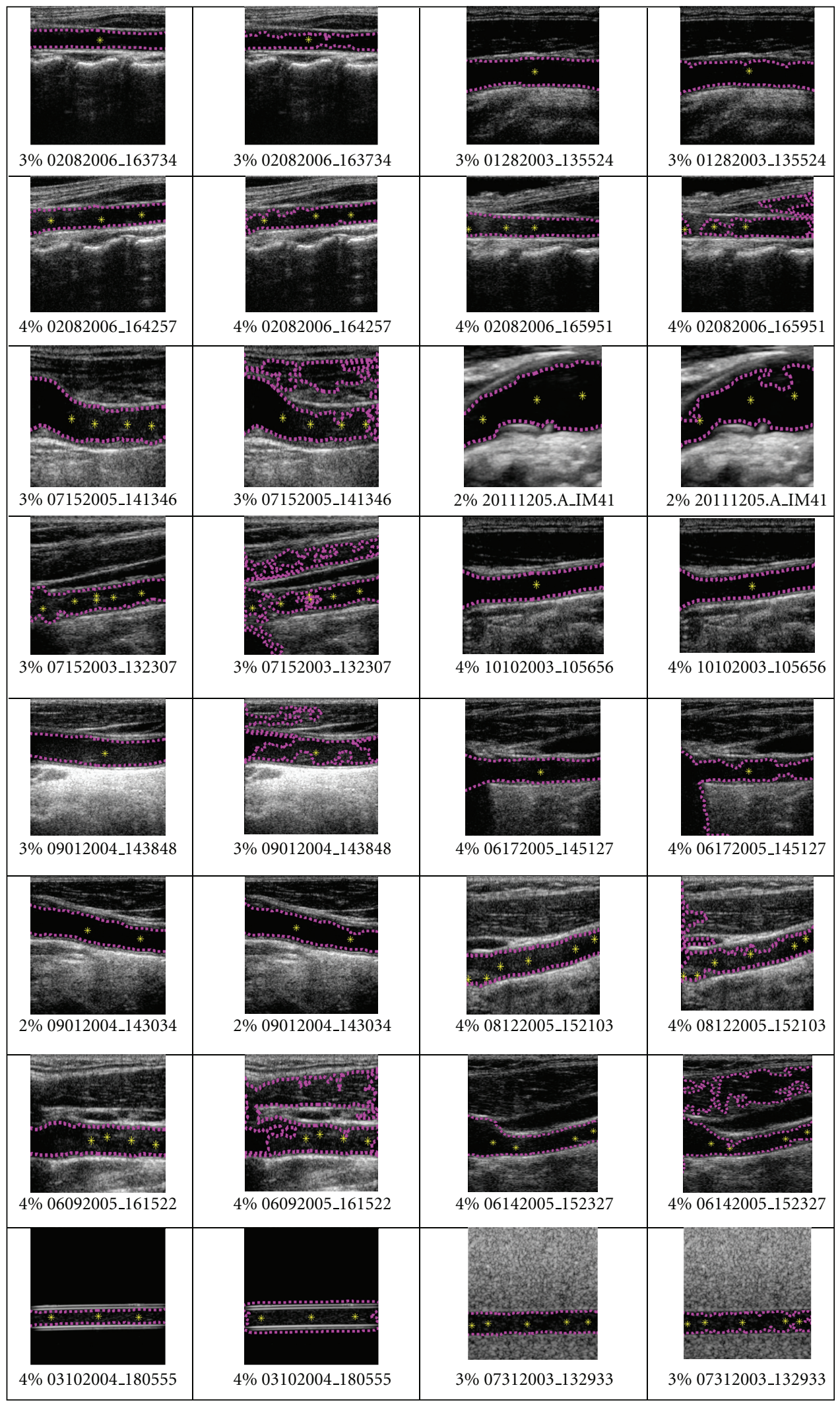

FIGURE 13: Comparison between the probabilistic algorithm (first and third columns) and conventional region growing based on intensity thresholding (second and fourth columns). Results are given in pairs and labels indicate file reference and threshold settings used. The two left-most figures on the bottom-most row are from the walled-flow phantom, and the two right-most figures on the same row are from the wall-less flow phantom. 
for the collection of the clinical data. They also thank Professor Thompson Robinson and Dr. Mark Horsfield for their kind support of this study. Funding is gratefully acknowledged from the National Institute for Health Research Collaboration in Applied Health Research and Care for Leicestershire, Northamptonshire, and Rutland (NIHR CLAHRC).

\section{References}

[1] S. Golemati, A. Sassano, M. J. Lever, A. A. Bharath, S. Dhanjil, and A. N. Nicolaides, "Carotid artery wall motion estimated from b-mode ultrasound using region tracking and block matching," Ultrasound in Medicine and Biology, vol. 29, no. 3, pp. 387-399, 2003.

[2] R. H. Selzer, W. J. Mack, P. L. Lee, H. Kwong-Fu, and H. N. Hodis, "Improved common carotid elasticity and intimamedia thickness measurements from computer analysis of sequential ultrasound frames," Atherosclerosis, vol. 154, no. 1, pp. 185-193, 2001.

[3] I. Wendelhag, Q. Liang, T. Gustavsson, and J. Wikstrand, "A new automated computerized analyzing system simplifies readings and reduces the variability in ultrasound measurement of intima-media thickness," Stroke, vol. 28, no. 11, pp. 2195-2200, 1997.

[4] R. E. Bellman and S. Dreyfus, Applied Dynamic Programming, Princeton University Press, Princeton, NJ, USA, 1962.

[5] F. Beux, S. Carmassi, M. V. Salvetti et al., "Automatic evaluation of arterial diameter variation from vascular echographic images," Ultrasound in Medicine and Biology, vol. 27, no. 12, pp. 1621-1629, 2001.

[6] D. C. Cheng, A. Schmidt-Trucksäss, K. S. Cheng, and H. Burkhardt, "Using snakes to detect the intimal and adventitial layers of the common carotid artery wall in sonographic images," Computer Methods and Programs in Biomedicine, vol. 67, no. 1, pp. 27-37, 2002.

[7] V. R. Newey and D. K. Nassiri, "Online artery diameter measurement in ultrasound images using artificial neural networks," Ultrasound in Medicine and Biology, vol. 28, no. 2, pp. 209-216, 2002.

[8] M. H. Cardinal, J. Meunier, G. Soulez, R. L. Maurice, E. Therasse, and G. Cloutier, "Intravascular ultrasound image segmentation: a three-dimensional fast-marching method based on gray level distributions," IEEE Transactions on Medical Imaging, vol. 25, no. 5, pp. 590-601, 2006.

[9] S. Golemati, J. Stoitsis, E. G. Sifakis, T. Balkizas, and K. S. Nikita, "Using the Hough transform to segment ultrasound images of longitudinal and transverse sections of the carotid artery," Ultrasound in Medicine and Biology, vol. 33, no. 12, pp. 1918-1932, 2007.

[10] G. Mendizabal-Ruiz, M. Rivera, and I. A. Kakadiaris, "A probabilistic segmentation method for the identification of luminal borders in intravascular ultrasound images," in Proceedings of the 26th IEEE Conference on Computer Vision and Pattern Recognition (CVPR '08), pp. 1-8, June 2008.

[11] X. Yang, M. Ding, L. Lou, M. Yuchi, W. Qiu, and Y. Sun, "Common carotid artery lumen segmentation in B-mode ultrasound transverse view images," International Journal of Image, Graphics and Signal Processing, vol. 5, pp. 15-21, 2011.

[12] J. A. Noble and D. Boukerroui, "Ultrasound image segmentation: a survey," IEEE Transactions on Medical Imaging, vol. 25, no. 8, pp. 987-1010, 2006.
[13] K. V. Ramnarine, B. Kanber, and R. B. Panerai, "Assessing the performance of vessel wall tracking algorithms: the importance of the test phantom," Journal of Physics, vol. 1, pp. 199-204, 2004.

[14] M. W. Claridge, G. R. Bate, J. A. Dineley et al., "A reproducibility study of a TDI-based method to calculate indices of arterial stiffness," Ultrasound in Medicine and Biology, vol. 34, no. 2, pp. 215-220, 2007.

[15] C. J. P. M. Teirlinck, R. A. Bezemer, C. Kollmann et al., "Development of an example flow test object and comparison of five of these test objects, constructed in various laboratories," Ultrasonics, vol. 36, no. 1-5, pp. 653-660, 1998.

[16] K. V. Ramnarine, D. K. Nassiri, P. R. Hoskins, and J. Lubbers, "Validation of a new blood-mimicking fluid for use in Doppler flow test objects," Ultrasound in Medicine and Biology, vol. 24, no. 3, pp. 451-459, 1998.

[17] K. V. Ramnarine, P. R. Hoskins, H. F. Routh, and F. Davidson, "Doppler backscatter properties of a blood-mimicking fluid for doppler performance assessment," Ultrasound in Medicine and Biology, vol. 25, no. 1, pp. 105-110, 1999.

[18] L. Germond, O. Bonnefous, and T. Loupas, "Quantitative assessment of the artery dilation measurements with an arterial phantom," in Proceedings of the 2001 Ultrasonics Symposium, pp. 1413-1416, October 2001.

[19] 2011, Region Growing (2D/3D greyscale). MATLAB Central File Exchange, http://www.mathworks.com/matlabcentral/ fileexchange/32532. 

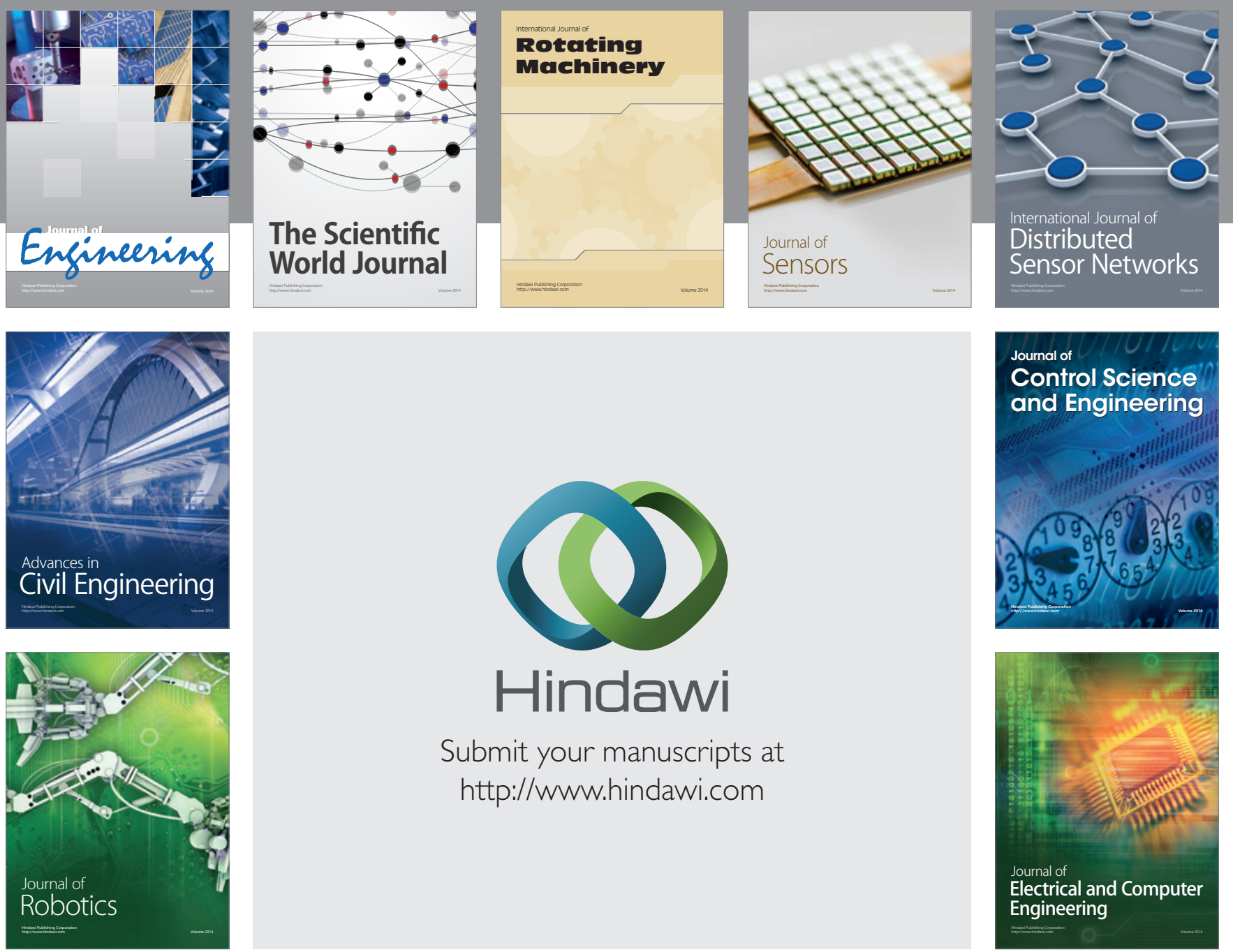

Submit your manuscripts at

http://www.hindawi.com
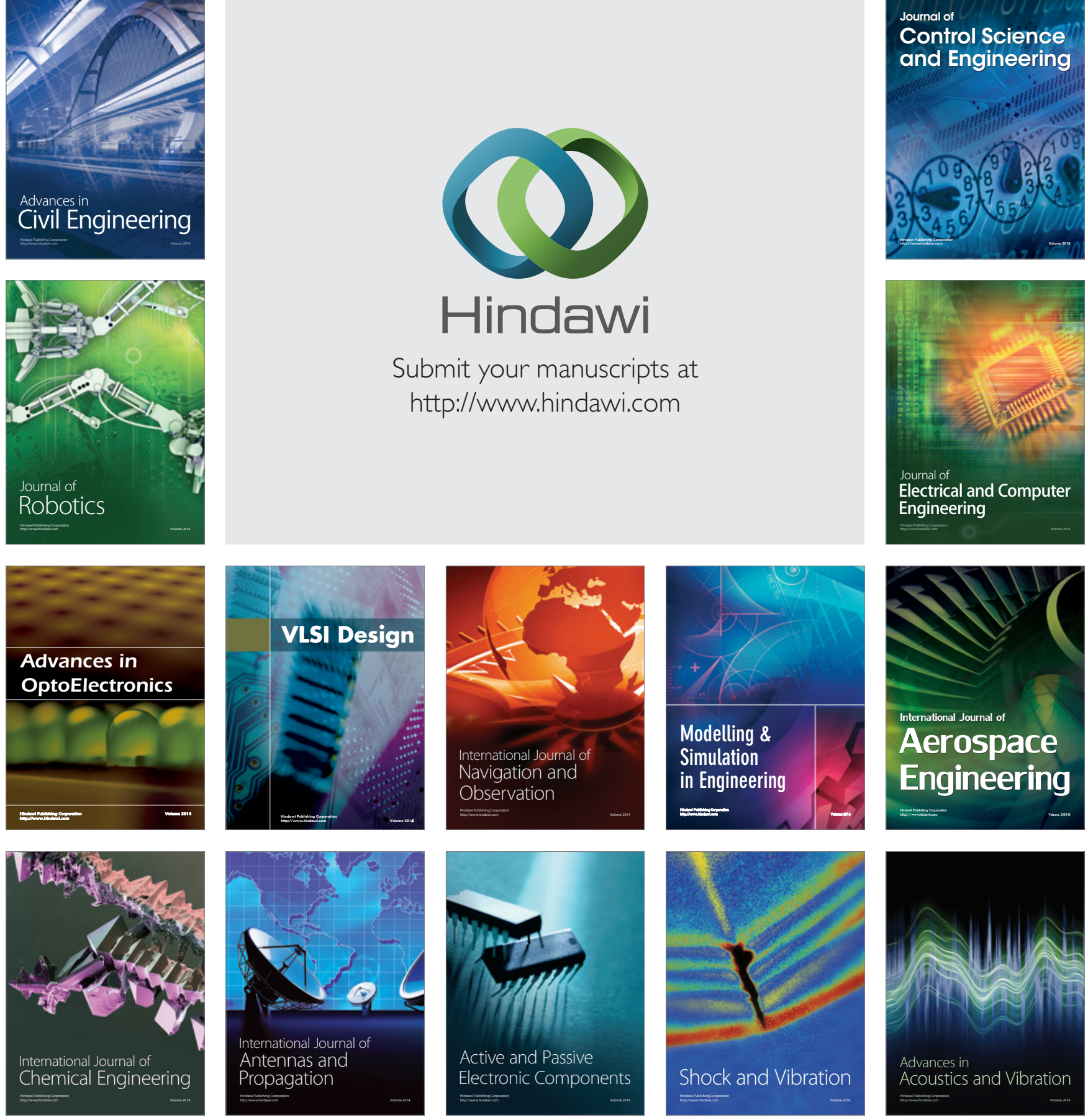